\title{
过硫酸铵氧化光度法测定微量锰的褪色机理探讨
}

\author{
上官恩波 ${ }^{1,2}$, 吴呈珂 ${ }^{2,}{ }^{*}$, 李晶 ${ }^{2}$, 冯素玲 2 , 李全民 ${ }^{2}$ \\ 1 河南师范大学材料科学与工程学院, 河南新乡 453003 \\ 2 河南师范大学化学化工学院, 河南 新乡 453003
}

摘要: 通过实验验证详细讨论了过硫酸铵氧化光度法测定微量锰的褪色机理, 结果表明: 过硫酸根离子受热分解所 产生的过氧化氢导致了 $\mathrm{MnO}_{4}^{-}$发生褪色反应, 用 $\mathrm{KIO}_{4}$ 代替过硫酸铵作为氧化剂测定微量锰不仅可以避免 $\mathrm{MnO}_{4}^{-}$发生褪 色现象, 还能获得准确测定结果。

关键词: 过硫酸铵; 微量锰; 裖色机理; 高碘酸钾

中图分类号: G64; 0657.32

\section{Study on the Discoloration Mechanism of Spectrophotometric Determination of Trace Manganese by Ammonium Persulfate Oxidation}

\author{
Enbo Shangguan ${ }^{1,2}$, Chengke $\mathrm{Wu}^{2,}{ }^{,}$, Jing $\mathrm{Li}^{2}$, Suling Feng ${ }^{2}$, Quanmin $\mathrm{Li}^{2}$ \\ ${ }^{1}$ School of Chemistry and Chemical Engineering, Henan Normal University, Xinxiang 453003, Henan Province, China. \\ ${ }^{2}$ School of Materials Science and Engineering, Henan Normal University, Xinxiang 453003, Henan Province, China.
}

\begin{abstract}
The discoloration mechanism of spectrophotometric determination of trace manganese by ammonium persulfate oxidation is discussed in detail through experimental verification. The results show that hydrogen peroxide produced by the thermal decomposition of supersulfate ions leads to the discoloration of permanganate ions, using $\mathrm{KIO}_{4}$ instead of ammonium persulfate as oxidant to determine trace manganese can not only avoid the fading phenomenon of $\mathrm{MnO}_{4}^{-}$, but also obtain accurate determination results.
\end{abstract}

Key Words: Ammonium persulfate; Trace manganese; Fading mechanism; Potassium periodate

过硫酸铵氧化分光光度法测定钢中锰至今已有 100 多年的历史 ${ }^{[1]}$, 该方法由于仪器价格低廉、 操作简便、分析成本低, 常常被基层检测机构用于生活饮用水中锰元素含量的测定 ${ }^{[2]}$ 。尽管电感耦 合等离子体质谱法和原子吸收光谱法具有灵敏度高、检测限低、可直接进行水样中锰元素含量的测 定等优点, 但由于仪器价格较贵以及运行和维护成本较高而不适宜基层检测机构普遍推广使用。由 于不同样品中锰元素经酸化处理后形成的 $\mathrm{Mn}(\mathrm{II})$ 能够被过硫酸铵在一定条件下氧化为紫红色的 $\mathrm{MnO}_{4}^{-}$, 从而使得这一经典分光光度法能够广泛应用于测定不同样品中锰元素的含量。该方法也是我 校基础仪器分析实验课程中测定钢样中微量锰的教学内容之一, 正是得益于这一实验教学内容的启

收稿: 2020-04-30; 录用: 2020-06-28; 网络发表：2020-07-21

“通讯作者, Email: ckwu0304@163.com

基金资助: 河南师范大学高等教育教学改革与实践(YJS2019JG09) 
发, 使得我们科研室在制备性能优良的锰酸锂电池正极材料以及废旧锰酸锂电池回收研究中, 也将 该方法应用于锰酸锂样品中锰含量的测定, 为研究工作的顺利进展提供了极大的便利。然而, 无论 是在多年的实验课教学中还是科研上对实际样品的测定中, 在对样品及标样中的 $\mathrm{Mn}(\mathrm{II})$ 用 $\left(\mathrm{NH}_{4}\right)_{2} \mathrm{~S}_{2} \mathrm{O}_{8}$ 加热煮沸进行氧化预处理时, 总是会时不常地出现生成的 $\mathrm{MnO}_{4}^{-}$紫红色发生完全褪色现 象。并且令人不解的是, 在 $\mathrm{MnO}_{4}^{-}$褪色后的溶液中再加入 $\left(\mathrm{NH}_{4}\right)_{2} \mathrm{~S}_{2} \mathrm{O}_{8}$ 进行重复氧化处理时, 该溶液并 不能再出现原来 $\mathrm{MnO}_{4}^{-}$的紫红色, 其原因究竟是什么? 因此, 探讨 $\left(\mathrm{NH}_{4}\right)_{2} \mathrm{~S}_{2} \mathrm{O}_{8}$ 氧化光度法测定微量 锰的褪色机理不仅对实际样品的测定, 而且对仪器分析实验课教学都具有一定的理论指导意义。

\section{$1\left(\mathrm{NH}_{4}\right)_{2} \mathrm{~S}_{2} \mathrm{O}_{8}$ 氧化光度法测定微量锰理论依据及显色反应存在的问题}

\section{1 理论依据}

根据文献 ${ }^{[3]}$, 当 $\mathrm{Ag}^{+}$存在时, 在溶液煮沸的温度条件下 $\mathrm{Mn}(\mathrm{II})$ 可被 $\left(\mathrm{NH}_{4}\right)_{2} \mathrm{~S}_{2} \mathrm{O}_{8}$ 氧化成紫红色的

$\mathrm{MnO}_{4}^{-}$, 其颜色的深浅(吸光度)与 $\mathrm{Mn}(\mathrm{II})$ 的浓度成正比。其显色反应为:

$$
5 \mathrm{~S}_{2} \mathrm{O}_{8}^{2-}+2 \mathrm{Mn}^{2+}+8 \mathrm{H}_{2} \mathrm{O}=2 \mathrm{MnO}_{4}^{-}+10 \mathrm{SO}_{4}^{2-}+16 \mathrm{H}^{+}
$$

\section{2 显色反应存在的问题}

根据上述显色反可知, 溶液中的 $\mathrm{Mn}(\mathrm{II})$ 能否完全被 $\left(\mathrm{NH}_{4}\right)_{2} \mathrm{~S}_{2} \mathrm{O}_{8}$ 氧化成紫红色的 $\mathrm{MnO}_{4}^{-}$是光度法 能否准确测定 $\mathrm{Mn}(\mathrm{II})$ 含量的关键。若 $\left(\mathrm{NH}_{4}\right)_{2} \mathrm{~S}_{2} \mathrm{O}_{8}$ 用量不足时 ${ }^{[4]}, \mathrm{Mn}(\mathrm{II})$ 不能完全被氧化成高价态的 $\mathrm{MnO}_{4}^{-}$, 易出现棕色(量大)或黑色(少量)中间价态的 $\mathrm{MnO}_{2}$ 沉淀, 影响结果的测定。因此, 通常在显色 反应中不仅需要加入过量的 $\left(\mathrm{NH}_{4}\right)_{2} \mathrm{~S}_{2} \mathrm{O}_{8}$, 同时又需要通过延长加热煮沸溶液的时间, 以保证 $\mathrm{Mn}(\mathrm{II})$ 能够定量被氧化为 $\mathrm{MnO}_{4}^{-}$。但正是由于加热煮沸进行显色反应的过程中, 形成 $\mathrm{MnO}_{4}^{-}$紫红色溶液又不 时地会出现褪色转变为无色溶液, 这与微量 $\mathrm{Mn}^{2+}$ 在溶液中呈现无色的现象相一致。这也表明 $\mathrm{MnO}_{4}^{-}$ 在受热分解时的产物是 $\mathrm{Mn}(\mathrm{II})$, 而不是通常所认为分解产物之一应该是出现黑色或棕色沉淀的 $\mathrm{MnO}_{2}$ 。可见, 加热煮沸进行显色反应时间长短对 $\mathrm{Mn}(\mathrm{II})$ 能否准确测定至关重要。加热时间不足时, 显色反应不完全，加热时间较长时，又可能导致生成的 $\mathrm{MnO}_{4}^{-}$紫红色发生褪色现象。

\section{$2\left(\mathrm{NH}_{4}\right)_{2} \mathrm{~S}_{2} \mathrm{O}_{8}$ 氧化光度法测定微量锰褪色机理探究及实验验证}

\section{1 褪色机理探究}

为了探究过硫酸铵氧化光度法测定微量锰的褪色机理, 在 $\mathrm{Ag}^{+}$和 $\left(\mathrm{NH}_{4}\right)_{2} \mathrm{~S}_{2} \mathrm{O}_{8}$ 存在下, 将含微量 $\mathrm{Mn}(\mathrm{II})$ 的溶液在红外光波炉 (控温 $200^{\circ} \mathrm{C}$ ) 者沸显色后, 再继续加热至 $\mathrm{MnO}_{4}^{-}$褪色、并自然冷却至室温。

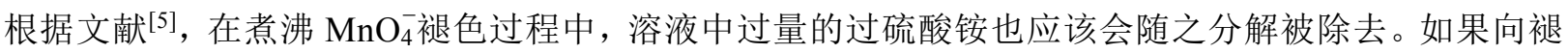
色后的溶液中再加入 $\mathrm{Ag}^{+}$和 $\left(\mathrm{NH}_{4}\right)_{2} \mathrm{~S}_{2} \mathrm{O}_{8}$ 进行煮沸重复处理时, 溶液中由 $\mathrm{MnO}_{4}^{-}$褪色形成的 $\mathrm{Mn}^{2+}$ 则不 会再被氧化为紫红色的 $\mathrm{MnO}_{4}^{-}$。这说明在上述褪色的溶液中可能存在有某种还原性组分, 从而抑制 了 $\mathrm{Mn}^{2+}$ 重新被氧化成 $\mathrm{MnO}_{4}^{-}$的化学反应。如果能够定性确定溶液中的还原性组分, 那么 $\mathrm{MnO}_{4}^{-}$在受 热分解时的产物正如前面所述是 $\mathrm{Mn}^{2+}$ 而不是棕色或黑色的 $\mathrm{MnO}_{2}$ 这个疑惑就能够迎刃而解。

根据文献 ${ }^{6]}$, 过二硫酸 $\left(\mathrm{H}_{2} \mathrm{~S}_{2} \mathrm{O}_{8}\right)$ 可以看作是过氧化氢 $\left(\mathrm{H}_{2} \mathrm{O}_{2}\right)$ 的衍生物, 过氧化氢中的两个氢原子 被 $\left(-\mathrm{HSO}_{3}\right)$ 取代。当过硫酸铵溶液受热分解时, 除了能分解为文献 ${ }^{[5]}$ 报道的 $\mathrm{HSO}_{4}^{-}$和 $\mathrm{O}_{2}$ 外, 有可能 还会产生 $\mathrm{H}_{2} \mathrm{O}_{2}$ 。可见可能正是 $\mathrm{S}_{2} \mathrm{O}_{8}^{2-}$ 分解产物产生的 $\mathrm{H}_{2} \mathrm{O}_{2}$, 才使得 $\mathrm{MnO}_{4}^{-}$在溶液中被 $\mathrm{H}_{2} \mathrm{O}_{2}$ 还原成 $\mathrm{Mn}^{2+}$ 而不是棕色或黑色 $\mathrm{MnO}_{2}$ 。其反应如下:

$$
\begin{aligned}
& \mathrm{S}_{2} \mathrm{O}_{8}^{2-}+2 \mathrm{H}_{2} \mathrm{O}=2 \mathrm{H}^{+}+2 \mathrm{SO}_{4}^{2-}+\mathrm{H}_{2} \mathrm{O}_{2} \\
& 2 \mathrm{MnO}_{4}^{-}+5 \mathrm{H}_{2} \mathrm{O}_{2}+6 \mathrm{H}^{+}=2 \mathrm{Mn}^{2+}+5 \mathrm{O}_{2}+8 \mathrm{H}_{2} \mathrm{O}
\end{aligned}
$$

\section{$2.2 \mathrm{~S}_{2} \mathrm{O}_{8}^{2-}$ 分解为 $\mathrm{H}_{2} \mathrm{O}_{2}$ 的定性鉴定及讨论}

为了定性检验 $\mathrm{S}_{2} \mathrm{O}_{8}^{2-}$ 的在煮沸时分解产物除了 $\mathrm{HSO}_{4}^{-}$和 $\mathrm{O}_{2}$ 外, 还会产生 $\mathrm{H}_{2} \mathrm{O}_{2}$, 不同实验设计方 案中溶液颜色变化及实验结果见表 1 。 
大学 化 学 Univ. Chem. 2021, 36 (4), 2004125 (3 of 4)

表 1 不同试验方法的溶液中颜色变化及结论

\begin{tabular}{|c|c|c|}
\hline 试验方法及溶液组成 & 实验操作与现象 & 结论 \\
\hline I. 总体积 $40 \mathrm{~mL}: 50 \mu \mathrm{g} \cdot \mathrm{mL}^{-1} \mathrm{Mn}^{2+} 、 1 \mathrm{~mol} \cdot \mathrm{L}^{-1} \mathrm{H}_{2} \mathrm{SO}$ 和 & 室温下 $\mathrm{Mn}^{2+}$ 不能被氧化为 & $\left(\mathrm{NH}_{4}\right)_{2} \mathrm{~S}_{2} \mathrm{O}_{8}$ 室温下没有氧化性 \\
\hline \multirow{2}{*}{$\mathrm{H}_{3} \mathrm{PO}_{4} 、 2 \mathrm{~g}\left(\mathrm{NH}_{4}\right)_{2} \mathrm{~S}_{2} \mathrm{O}_{8} 、 0.3 \mathrm{~mL} 0.1 \mathrm{~mol} \cdot \mathrm{L}^{-1} \mathrm{AgNO}_{3}$} & $\mathrm{MnO}_{4}^{-}$ & \\
\hline & 加入 KI-淀粉溶液不变蓝 & \\
\hline II. 将 I 加热煮沸产生紫红色 $\mathrm{MnO}_{4}^{-}$再继续加热至溶液完 & 加入 KI-淀粉, 溶液变蓝 & 溶液中有 $\mathrm{H}_{2} \mathrm{O}_{2}$ 生成, 既使 $\mathrm{MnO}_{4}^{- \text {褪色 }}$ \\
\hline 全褪至无色, 冷却至室温 & & 又使淀粉变蓝, 见化学反应(2-4) \\
\hline III. 取 $2 \mathrm{~mL}$ 溶液 II 于 $10 \mathrm{~mL}$ 离心管中, 加入少许 & 在沸水浴加热数分钟, 溶液中出 & $\mathrm{Cr}_{2} \mathrm{O}_{7}^{2-}$ 被 $\mathrm{H}_{2} \mathrm{O}_{2}$ 氧化为蓝色 $\mathrm{CrO}_{5}$ 又快速 \\
\hline $\mathrm{K}_{2} \mathrm{Cr}_{2} \mathrm{O}_{7}$, 使其溶解, 溶液呈浅黄色 & 现蓝色 $\left(\mathrm{CrO}_{5}\right)$ 后又立即消失 & 分解见化学反应 $(5,6)$ \\
\hline \multirow[t]{2}{*}{ IV. 向溶液 II 再加入 $2 \mathrm{~g}\left(\mathrm{NH}_{4}\right)_{2} \mathrm{~S}_{2} \mathrm{O}_{8}$} & 加热至沸数分钟, 溶液中不再出 & 表明 $\mathrm{Mn}^{2+}$ 不能再被氧化, 溶液中有 \\
\hline & 现 $\mathrm{MnO}_{4}^{-}$的紫红色 & $\mathrm{H}_{2} \mathrm{O}_{2}$ 生成 \\
\hline V. 室温下总体积 $40 \mathrm{~mL}: 50 \mu \mathrm{g} \cdot \mathrm{mL}^{-1} \mathrm{Mn}^{2+}+$ & 加热, $\mathrm{Mn}^{2+}$ 立即被氧化 $\mathrm{MnO}_{4}^{-}$, 继 & 在热溶液中 $\mathrm{MnO}_{4}^{-}$没有被还原 $\mathrm{Mn}^{2+}$ \\
\hline $1 \mathrm{~mol} \cdot \mathrm{L}^{-1} \mathrm{H}_{2} \mathrm{SO}_{4}+1 \mathrm{~mol} \cdot \mathrm{L}^{-1} \mathrm{H}_{3} \mathrm{PO}_{4}+1 \mathrm{~g} \mathrm{KIO} 4$ & 续煮沸 $30 \mathrm{~min}$, 颜色仍不变 & \\
\hline
\end{tabular}

从表 1 中可以看出, 在室温下“溶液 I”为无色, 也不能使 $\mathrm{KI}$-淀粉溶液变蓝, 表明室温下 $\left(\mathrm{NH}_{4}\right)_{2} \mathrm{~S}_{2} \mathrm{O}_{8}$ 不具有氧化性; 若向加热使紫红色 $\mathrm{MnO}_{4}^{-}$褪去的 “溶液 II” 中加入 $\mathrm{KI}$-淀粉溶液, 溶液又立即变蓝, 这种紫红色褪去和溶液变蓝分别与所发生的化学反应(3)和(4)颜色变化相一致, 进一步证实了 $\mathrm{S}_{2} \mathrm{O}_{8}^{2-}$ 在煮沸条件下除了能分解为 $\mathrm{HSO}_{4}^{-}$和 $\mathrm{O}_{2}$ 外, 还有既有能使 $\mathrm{MnO}_{4}^{-}$还原为 $\mathrm{Mn}(\mathrm{II})$ 褪色又能使碘离子 $\left(\mathrm{I}^{-}\right)$ 氧化的 $\mathrm{H}_{2} \mathrm{O}_{2}$ 生成。

$$
2 \mathrm{I}^{-}+\mathrm{H}_{2} \mathrm{O}_{2}+2 \mathrm{H}^{+}=\mathrm{I}_{2}+2 \mathrm{H}_{2} \mathrm{O}
$$

为了进一步证明 $\mathrm{S}_{2} \mathrm{O}_{8}^{2-}$ 的分解产物除了 $\mathrm{HSO}_{4}^{-}$和 $\mathrm{O}_{2}$ 外还有 $\mathrm{H}_{2} \mathrm{O}_{2}$, 按照表 1 中 “溶液 III” 的试验 方法, 结果观察到溶液出现蓝色现象后又很快消失。根据文献 ${ }^{[5]}$, 在强酸性溶液中 $\mathrm{Cr}_{2} \mathrm{O}_{7}^{2-}$ 可以被 $\mathrm{H}_{2} \mathrm{O}_{2}$ 氧化生成蓝色的过氧化铬 $\mathrm{CrO}_{5}$ 或其水合物过铬酸 $\mathrm{H}_{2} \mathrm{CrO}_{6}$, 如化学反应(5), 而 $\mathrm{CrO}_{5}$ 在水中又极不稳 定而分解见化学反应(6)。同样也表明了在 $\mathrm{MnO}_{4}^{-}$裉色后的 “溶液 II” 中有能够使 $\mathrm{Cr}_{2} \mathrm{O}_{7}^{2-}$ 氧化为蓝色 过氧化铬 $\mathrm{CrO}_{5}$ 的 $\mathrm{H}_{2} \mathrm{O}_{2}$ 生成。

$$
\begin{aligned}
& \mathrm{Cr}_{2} \mathrm{O}_{7}^{2-}+4 \mathrm{H}_{2} \mathrm{O}_{2}+2 \mathrm{H}^{+}=\mathrm{CrO}_{5} \text { (蓝色) }+5 \mathrm{H}_{2} \mathrm{O} \\
& \mathrm{CrO}_{5}+12 \mathrm{H}^{+}=4 \mathrm{Cr}^{3+}+6 \mathrm{H}_{2} \mathrm{O}
\end{aligned}
$$

从表 1 中 “试验方法 $\mathrm{IV}$ ” 的实验现象又可以看出, 由 $\mathrm{MnO}_{4}^{-}$褪色形成的 $\mathrm{Mn}^{2+}$ 不能被加入的 $\left(\mathrm{NH}_{4}\right)_{2} \mathrm{~S}_{2} \mathrm{O}_{8}$ 再次氧化为 $\mathrm{MnO}_{4}^{-}$, 这可能是由于 “溶液 II” 中有 $\mathrm{H}_{2} \mathrm{O}_{2}$ 存在时, 它诱导了 $\mathrm{S}_{2} \mathrm{O}_{8}^{2-}$ 发生上 述化学反应(2), 而不是发生 $\mathrm{S}_{2} \mathrm{O}_{8}^{2-}$ 将 $\mathrm{Mn}^{2+}$ 氧化为 $\mathrm{MnO}_{4}^{-}$的化学反应(1)。

从表 1 “溶液 V” 中还可以看出, 当用 $\mathrm{KIO}_{4}$ 取代 $\left(\mathrm{NH}_{4}\right)_{2} \mathrm{~S}_{2} \mathrm{O}_{8}$ 和 $\mathrm{AgNO}_{3}$ 时, 结果发现, 当 $\mathrm{Mn}(\mathrm{II})$ 被氧化成紫红色的 $\mathrm{MnO}_{4}^{-}$后, 继续加热煮沸 $30 \mathrm{~min}$ (不时地补充去离子水), 其颜色也不会因煮沸时 间延长而裉色。这是由于在过量强氧化剂 $\mathrm{KIO}_{4}$ 存在下, 始终使 $\mathrm{Mn}^{2+}$ 处于被氧化为 $\mathrm{MnO}_{4}^{-}$氧化态, 而不是像 $\mathrm{S}_{2} \mathrm{O}_{8}^{2-}$ 那样受热分解会产生具有使 $\mathrm{MnO}_{4}^{-}$还原的 $\mathrm{H}_{2} \mathrm{O}_{2}$ 。可见, 尽管在一定条件下 $\left(\mathrm{NH}_{4}\right)_{2} \mathrm{~S}_{2} \mathrm{O}_{8}$ 也具有很强的氧化性, 但氧化剂的分子结构对氧化产物稳定性的影响也是至关重要的。正是由于 $\mathrm{S}_{2} \mathrm{O}_{8}^{2-}$ 离子结构中含有过氧键 $\left(-\mathrm{O}-\mathrm{O}-\right.$ ) 受热分解生成了 $\mathrm{H}_{2} \mathrm{O}_{2}$, 才导致发生了将 $\mathrm{MnO}_{4}^{-}$还原为 $\mathrm{Mn}^{2+}$ 的副反应。因此, 使用 $\mathrm{KIO}_{4}$ 作为光度法测定微量 $\mathrm{Mn}(\mathrm{II})$ 的氧化显色剂, 可以保证测定结果的可靠性 和准确性。

\section{$3 \mathrm{KIO}_{4}$ 氧化光度法测定微量 $\mathrm{Mn}(\mathrm{II})$}

以工作曲线制作为例: 用移液管分别吸取 $0.5 \mathrm{mg} \cdot \mathrm{mL}^{-1} \mathrm{Mn}(\mathrm{II})$ 标准溶液 $1.00 、 2.00 、 3.00 、 4.00 \mathrm{~mL}$ 于 $50 \mathrm{~mL}$ 烧杯中, 分别加入 $2.5 \mathrm{~mL}$ 浓 $\mathrm{H}_{2} \mathrm{SO}_{4}$ 和 $2.5 \mathrm{~mL} 85 \%$ 的 $\mathrm{H}_{3} \mathrm{PO}_{4}$, 将溶液稀释至约 $40 \mathrm{~mL}$, 加 入 $1 \mathrm{~g} \mathrm{KIO}_{4}$, 加热至沸约 $10 \mathrm{~min}$, 冷却, 将溶液转入 $50 \mathrm{~mL}$ 容量瓶中, 稀释至刻度, 摇匀。以去离子 
水空白为参比, 于 $545 \mathrm{~nm}$ 波长处测定吸光度, 绘制工作曲线。

\section{4 结语}

从上述讨论可知, 本文依据设计试验中溶液颜色变化和所对应的化学反应之间的关系, 从理论 和实验上揭示了过硫酸铵氧化光度法测定微量锰的裉色的原因。这无疑对提高实验课教学水平具有 一定的理论指导意义。尤其是在光度法测定微量 $\mathrm{Mn}(\mathrm{II})$ 时, 用 $\mathrm{KIO}_{4}$ 代替 $\left(\mathrm{NH}_{4}\right)_{2} \mathrm{~S}_{2} \mathrm{O}_{8}$, 不仅不需要外 加催化剂, 也不会因加热煮沸时间不当出现 $\mathrm{Mn}(\mathrm{II})$ 没有完全被氧化或被氧化为 $\mathrm{MnO}_{4}^{-}$后又出现裉色 现象, 从而避免了原实验方法中对加热煮沸时间控制较为严格的苛刻要求, 为光度法简便、快速、准 确测定微量锰含量提供了保证。

\section{参 考 文 献}

[1] 赵国城. 分析试验室, 1984, 3 (2), 37.

[2] 王明侠, 丁恒. 环境与健康杂志, 2012, 29 (10), 947.

[3] 刘志滨, 韩砚齐, 吴维. 供水技术, 2009, 3(1), 51.

[4] 张福梅, 李响. 中国金属通报, 2009, No. 4, 274.

[5] 武汉大学, 吉林大学, 中国科学技术大学, 中山大学, 南开大学 编. 分析化学. 第 1 版. 北京: 人民教育出版社, 1978: 284, 40.

[6] Nebergall, W. H.; Schmidt, F. C.; Holclaw, H. F. 普通化学(第三分册). 马维, 等译. 北京: 人民教育出版社, 1980: 110. 